\title{
DifFerential Evolution as Calibration TECHNIQUE FOR THREE AXIS GYROSCOPE
}

\author{
Ales Kuncar, Martin Sysel \& Tomas Urbanek
}
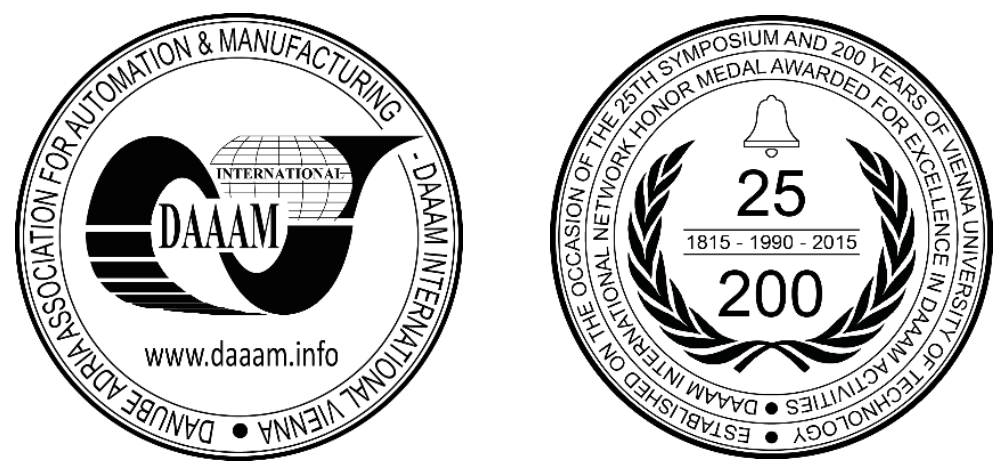

This Publication has to be referred as: Kuncar, A[les]; Sysel, M[artin] \& Urbanek, T[omas] (2017). Differential Evolution as Calibration Technique for Three Axis Gyroscope, Proceedings of the 28th DAAAM International Symposium, pp.0592-0596, B. Katalinic (Ed.), Published by DAAAM International, ISBN 978-3-902734-11-2, ISSN 1726-9679, Vienna, Austria

DOI: $10.2507 / 28$ th.daaam.proceedings.083

\begin{abstract}
The MEMS (Micro-Electro-Mechanical Systems) gyroscopes are widely used in many engineering applications and areas. These types of sensors are small, cheap, and have low power consumption; however, these sensors are not entirely accurate. They are influenced by many factors such as sensor errors (scale factor and bias) and manufacturing errors (misalignment); therefore, the calibration of the gyroscope is necessary before its application in systems. Thus, this research paper describes calibration technique for three axis gyroscope. The differential evolution (DE) algorithm is used for the determination of the scale factor, misalignment, and bias. The performance of this method is analysed in experiment on three axis low-cost gyroscope L3GD20.
\end{abstract}

Keywords: Calibration; Differential Evolution; Gyroscope; MEMS; Sensors

\section{Introduction}

Three axis gyroscopes are sensors that measure angular velocity (rotations in general) around each sensitive axis. MEMS gyroscopes use the principle of Coriolis force of the moving object. Coriolis force can be formulated as

$$
F=2 \cdot m \cdot \vec{\omega} \times \vec{v}
$$

where $m$ is the mass of the object, $\vec{\omega}$ is the angular velocity vector and $\vec{v}$ is the velocity with respect to the rotating system.

These sensors, along with accelerometers, are one of the most used sensors for inertial navigation systems [1][2] and motion sensing [3][4]. Gyroscopes are furthermore used in many areas and applications such as guidance systems, assistance of stability (bicycles, segway, etc.). However, these sensors based on MEMS technology are vulnerable to systematic errors (scale factor, bias and misalignment). Therefore, there is more emphasis on the calibration of the sensor.

The biggest error source, that has an impact on the accuracy of the measurements, is bias. This error can be simply minimized by stationary readings and then averaging these values [5]. To minimize the scale factor and misalignment angles, special equipment (turntable) is required. Olivares et al. [6] proposed a calibration technique using accelerometer to aid calibrate the gyroscope because the accelerometers are easier to calibrate and more accurate. On the other hand, 
Wu et al. [7] used magnetometer instead of accelerometer. This method requires a sufficient number of rotations to cover the local magnetic field. Then these measurements can be used as a reference for calibration of gyroscope.

The reminder of this paper is organized as follows. In section 2, the differential evolution is introduced. The gyroscope error model and used equipment are briefly described in section 3 and section 4, respectively. The gyroscope calibration and its details are mentioned in section 5 .

\section{Differential Evolution}

Differential evolution is an optimization heuristic algorithm which search the global minimum or maximum introduced by R. Storn and K. Price in 1995 [8]. This optimization method is an evolutionary algorithm based on population, mutation, and recombination.

The algorithm uses a mutation and recombination as a search mechanism and a selection that leads the search to perspective areas in the search space. The DE algorithm uses a recombination operator which can choose child parameters from one parent more often than from others. A recombination effectively shuffles information about successful combinations by the use of members from existing population allowing the search for a better solution space.

The optimization tasks consist of D parameters that represent a D-dimensional vector. The initial population of NP solutions is created randomly. Then the population is going to be adjusted using mutation, recombination and selection operators.

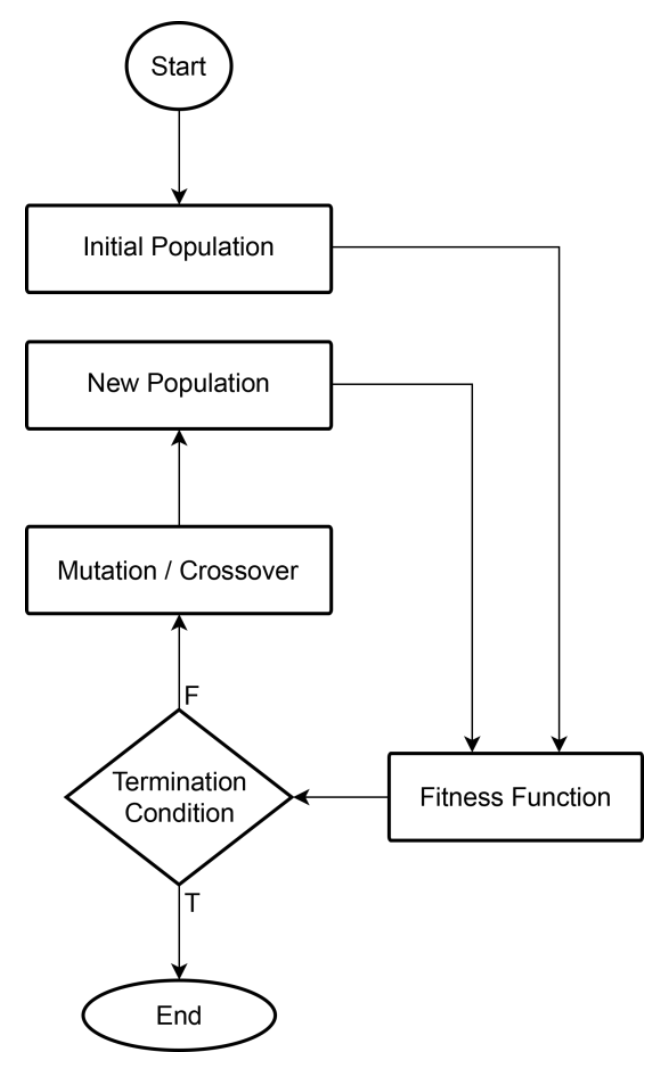

Fig. 1. Flow-chart of DE for parameters estimation.

Differential evolution uses only four parameters; therefore, it is easy to implement. Parameters are Generations, NP, F, and CR [9].

- Generations (Number of iterations) specifies the number of evolutionary cycles (generations) during which the entire population develops.

- NP (Number of population members) is a parameter which gives the size of the population. The value of this parameter cannot be lower than 4 because it is the minimum size at which the differential evolution algorithm still works. The optimal set up of this parameter is $5 \cdot \mathrm{D}<=\mathrm{NP}<=10 \cdot \mathrm{D}[8]$, where $\mathrm{D}$ is dimension of the problem.

- $\mathrm{CR}$ (Crossover probability). This parameter is a small value in range from 0 to 1 . In case of separable function, this value is set close to 0 (clean copy of the fourth parent). Otherwise, it is set to the values close to 1 (random search).

- F (Mutation constant) is the last control parameter for differential evolution and its value ranges from 0 to 2 . This parameter produces variability of the population.

The flow chart of differential evolution algorithm for the calibration parameters estimation is shown in Fig. 1. 


\section{Gyroscope Error Model}

Sensor errors negatively influence the measurement results, thereby causing ambiguity results. Errors are caused by influences of the external environment, as well as internal influences. These errors can be divided up onto systematic and stochastic. Systematic errors can be reduced by calibration to remove their impact on measurements.

Measured values of three axis gyroscopes are affected by many error sources. These errors lead to a continuous increase in the position errors due to integration.

Mathematical model, that describes the measurements of the gyroscope, is expressed

$$
\left|\begin{array}{c}
\omega_{X} \\
\omega_{Y} \\
\omega_{Z}
\end{array}\right|=\left|\begin{array}{ccc}
S_{X} & 0 & 0 \\
\alpha & S_{Y} & 0 \\
\beta & \gamma & S_{Z}
\end{array}\right| \cdot\left|\begin{array}{c}
M_{X} \\
M_{Y} \\
M_{Z}
\end{array}\right|+\left|\begin{array}{c}
O_{X} \\
O_{Y} \\
O_{Z}
\end{array}\right|
$$

where $\omega_{X}, \omega_{Y}$, and $\omega_{Z}$ are calibrated gyroscope values. The coefficients $S_{X}, S_{Y}$, and $S_{Z}$ and $O_{X}, O_{Y}$, and $O_{Z}$ represent scale factor error, and bias, respectively. The angles $\alpha, \beta$, and $\gamma$ compensates the misalignment of the sensor axis. The $M_{X}, M_{Y}$, and $M_{Z}$ coefficients in the matrix are the measured values.

- Scale Factor - Scale factor is defined as the ratio between digital output and mechanical input of the gyroscope, i.e. it represents the slope of the function of the sensor. Ideally, the scale factor should be 1, so any difference from 1 is affecting the sensor. The difference between the ideal and the actual value is a sensitivity error, which is referred to as the scale factor error.

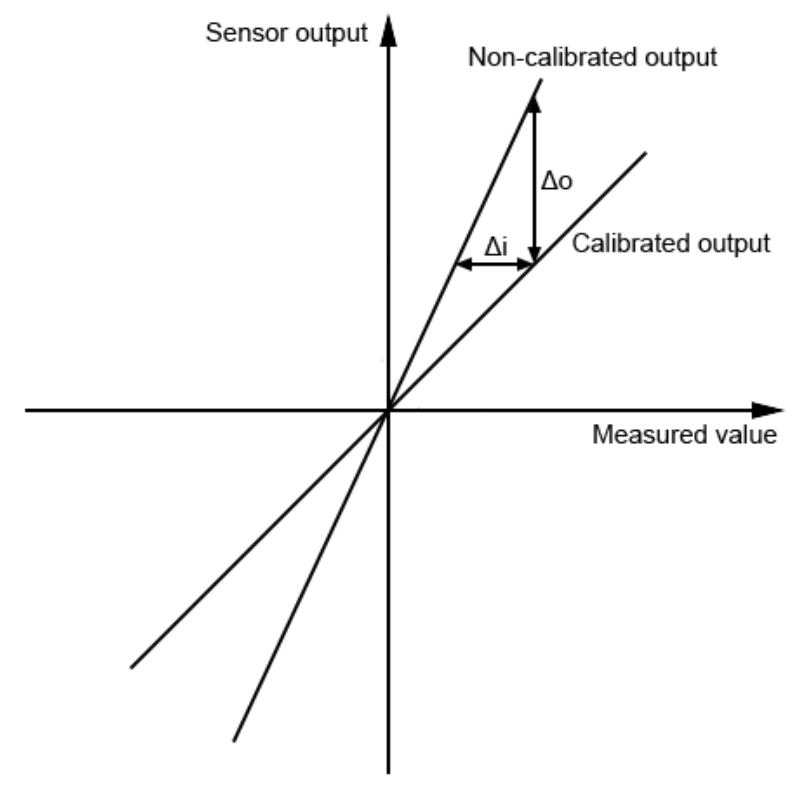

Fig. 2. Scale factor error.

- Misalignment error - This is an error in slight rotation of the sensor relative to the reference position. It is represented as angles between the sensor axis and the device body axis. This caused by imperfect mounting of sensor on the PCB (printed circuit board).

- Bias - If no external forces are applied on the gyroscope, its output should ideally show a zero value. However, there is an offset (zero shift), referred to as bias.

\section{Equipment}

The experimental measuring set (Fig. 3. Measurement chain.Fig. 3) includes a control unit, an inertial measurement unit (IMU) and software for data collection.

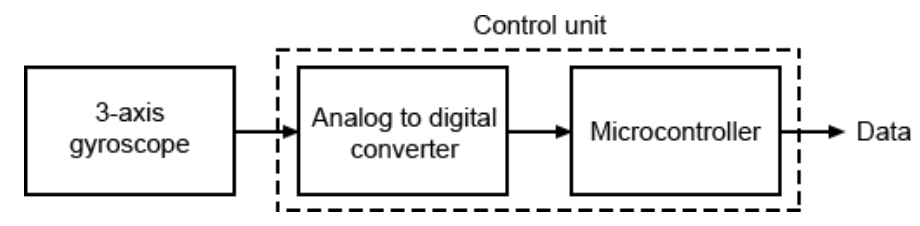

Fig. 3. Measurement chain. 
The STEVAL-MKI109V2 motherboard/control unit is designed to provide a platform for the evaluation of MEMS inertial modules. These modules can be connected via a 24-pin expansion socket.

The unit also includes a 512kB high performance ARM-based 32bit STM32F103RET6 microcontroller, which acts as a bridge between MEMS and GUI, or dedicated software routines for customized applications.

For measurements, the STEVAL-MKI124V1 10-axis inertial measurement unit is connected to the control unit. The IMU includes a three-axis gyroscope with an internal thermometer (L3GD20), a three-axis accelerometer and a three-axis magnetometer (LSM303DLHC) and a barometer (LPS331AP). All these sensors are based on MEMS technology and are factory tested and trimmed. However, this factory calibration is only suitable for basic applications. Advanced calibration has to be provided for applications such as navigation systems.

Several different configurations allow specific usage settings. Sensor specifications are given in Table 1. and [10].

\begin{tabular}{|c|c|}
\hline Parameters & Values \\
\hline Full scale & $250-2000 \mathrm{dps}$ \\
\hline Sensitivity & $8.75-70 \mathrm{mdps} / \mathrm{LSB}$ \\
\hline Min. zero level & $\pm 10- \pm 75 \mathrm{dps}$ \\
\hline Zero level vs. temp & $\pm 0.03- \pm 0.04 \mathrm{dps} /{ }^{\circ} \mathrm{C}$ \\
\hline Noise density & $0.03 \mathrm{dps} / \sqrt{\mathrm{Hz}}$ \\
\hline Linearity & $0.2 \% \mathrm{FS}$ \\
\hline
\end{tabular}

Table 1. Gyroscope Characteristics

To collect the measured data, the PC is connected to the control unit via a virtual serial port. The drivers for interaction and configuration of sensors are installed on the computer. This software is called Unico STSW-MKI109.

The collected data are pre-processed in Wolfram Mathematica 10 and then the differential evolution algorithm, programmed in Lua, is applied.

\section{Gyroscope Calibration}

The main objective of calibration is to reduce the errors, especially systematic errors (scale factor, bias). For this purpose, several measurements are needed in known conditions, in other words, the necessary data to determine the correction parameters.

\subsection{Fitness Function}

The fitness function is based on the sum of the squared errors where the error is the difference between the calculated output from current parameters and the true value. Therefore, the fitness function of the DE algorithm is modelled as

$$
F=\sum_{i=1}^{n}(X+Y+Z-\Omega)^{2}
$$

where $X, Y$, and $Z$ are calibrated values and $\Omega$ is the true value of angular velocity.

\subsection{Set-up of Differential Evolution}

The set-up of differential evolution is shown in Table 2. The parameter NP is set to the upper limitation suggested by [8]. That means to $10 \cdot \mathrm{D}$ where $\mathrm{D}$ represents the dimension of the problem (the number of unknown calibration parameters). In this case the dimension is 9 . The number of generations is given by the experiment that showed the optimal value around 300 (slow convergence rate to minimum). The parameters $\mathrm{F}$ and $\mathrm{CR}$ were also determined empirically.

\begin{tabular}{|c|c|}
\hline Parameter & Value \\
\hline NP & 90 \\
\hline Generations & 300 \\
\hline F & 0.5 \\
\hline CR & 0.7 \\
\hline
\end{tabular}

Table 2. Set-up of differential evolution 


\section{Conclusion}

The low-cost MEMS gyroscopes suffer from various sources of errors. In order to improve the accuracy of the measurements, the calibration of the sensor is necessary. Therefore, we presented calibration method for three axis gyroscope using differential evolution algorithm which compensates scale factor error, misalignment angles, and bias. For this method, simple turntable was used. The calibration was tested and analysed on gyroscope L3GD20. In the future work, we are going focus on sensor fusion using Kalman filters.

\section{Acknowledgments}

This work was supported by Internal Grant Agency of Tomas Bata University in Zlin under the project No. IGA/FAI/2017/007.

\section{References}

[1] Marinushkin, P.; Levitskiy, A. \& Zograf, F. (2017) Improving pedestrian navigation system performance through the use of non-orthogonal redundant inertial measurement units, in 2017 International Siberian Conference on Control and Communications (SIBCON), pp. 1-5.

[2] Abdulrahim, K.; Hide, C.; Moore, T. \& Hill, C. (2010). Aiding MEMS IMU with building heading for indoor pedestrian navigation, in 2010 Ubiquitous Positioning Indoor Navigation and Location Based Service, UPINLBS 2010, 2010.

[3] Galka, J.; Masior, M.; Zaborski, M. \& Barczewska, K. (2016). Inertial Motion Sensing Glove for Sign Language Gesture Acquisition and Recognition, IEEE Sens. J., vol. 16, no. 16, pp. 6310-6316, Aug. 2016.

[4] Bardyn, F.; Savary, M.; Grassi, S.; Farine, P.-A.; Fasel, B. \& Aminian, K. (2016). MEMS Inertial Motion Sensing Watch for Measuring Walking and Running Activities, in 2016 IEEE International Workshop on Signal Processing Systems (SiPS), 2016, pp. 104-109.

[5] Looney, M. (2010). A simple calibration for MEMS gyroscopes, Analog Devices, pp. 28-31, Jul-2010.

[6] Olivares, A.; Olivares, G.; Gorriz, J. M. \& Ramirez, J. (2009). High-efficiency low-cost accelerometer-aided gyroscope calibration, in 2009 International Conference on Test and Measurement, 2009, pp. 354-360.

[7] Wu, Y. \& Pei, L. (2017), Gyroscope Calibration via Magnetometer, IEEE Sens. J., vol. 17, no. 16, pp. 5269-5275, Aug. 2017.

[8] Storn, R. \& Price, K. (1995). Differential Evolution - A simple and efficient adaptive scheme for global optimization over continuous spaces.

[9] Storn, R. (1996). On the usage of differential evolution for function optimization, in Proceedings of North American Fuzzy Information Processing, pp. 519-523.

[10] STMicroelectronics (2013), Data brief: STEVAL-MKI124V1. p. 4. 